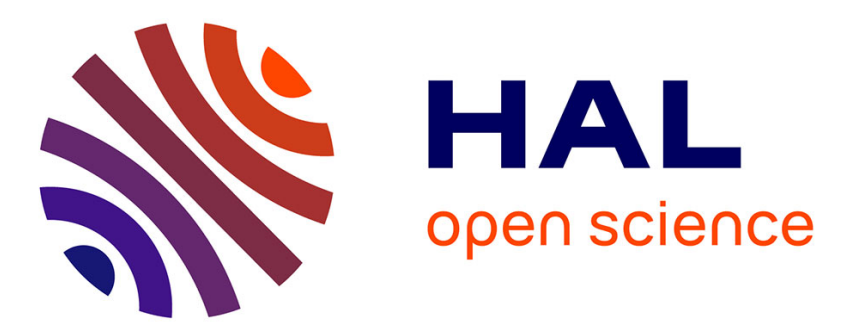

\title{
Polyamorphism of a Ce-based bulk metallic glass by high-pressure and high-temperature density measurements
}

\author{
F. Decremps, Guillaume Morard, G. Garbarino, M. Casula
}

\section{To cite this version:}

F. Decremps, Guillaume Morard, G. Garbarino, M. Casula. Polyamorphism of a Ce-based bulk metallic glass by high-pressure and high-temperature density measurements. Physical Review B: Condensed Matter and Materials Physics (1998-2015), 2016, 93 (5), pp.054209. 10.1103/PhysRevB.93.054209 . hal-01289155

\section{HAL Id: hal-01289155 \\ https://hal.science/hal-01289155}

Submitted on 5 Apr 2016

HAL is a multi-disciplinary open access archive for the deposit and dissemination of scientific research documents, whether they are published or not. The documents may come from teaching and research institutions in France or abroad, or from public or private research centers.
L'archive ouverte pluridisciplinaire HAL, est destinée au dépôt et à la diffusion de documents scientifiques de niveau recherche, publiés ou non, émanant des établissements d'enseignement et de recherche français ou étrangers, des laboratoires publics ou privés. 


\title{
Polyamorphism of a Ce-based bulk metallic glass by high-pressure and high-temperature density measurements.
}

\author{
F. Decremps ${ }^{a}$, G. Morard $^{a}$, G. Garbarino ${ }^{b}$, and M. Casula ${ }^{a}$ \\ a IMPMC, Université Pierre et Marie Curie, 75252 Paris, France. \\ b ESRF, F-38043 Grenoble, France
}

(Dated: March 30, 2016)

\begin{abstract}
Metallic glasses are of recent interest worldwide due to their remarkable physico-chemical properties which can be put in relation with their crystalline counterparts. Among them, cerium based metallic glasses (Ce-MGs) have unique features such as the existence of polyamorphism under pressure, unexpected in these spatially compact systems. While a phase transition between amorphous phases with change of density and local structure has been previously detected, the corresponding structural variation under pressure was not clearly identified, due to difficulties in performing accurate measurements and reliable analysis. In this work, angle dispersive x-ray diffraction experiments of $\mathrm{Ce}_{69} \mathrm{Al}_{10} \mathrm{Cu}_{20} \mathrm{Co}_{1}$ bulk metallic glass have been performed up to $16 \mathrm{GPa}$ along two distinct isotherms (300 and $340 \mathrm{~K}$ ). The whole diffuse signals have then been processed in order to extract the structure factor $S(Q)$, the pair distribution $g(r)$, the atomic density $\rho$ and the compressibility as a function of pressure and temperature. These are crucial probes to fully characterize the phase diagram, and they clearly confirm the existence of a link between polyamorphism in Ce-MGs and the $\gamma \leftrightarrows \alpha$ transition in pure cerium. Finally, owing to the presence of a critical point in pure solid Ce, the existence of such a feature is here discussed for Ce-MGs.
\end{abstract}

PACS numbers: 61.43.Fs,64.70.kj, 81.30.Hd 


\section{INTRODUCTION}

Bulk metallic glasses (BMG) are among the most interesting amorphous materials which combine the electric conductivity of metals with glassy structural disorder, thus promising to be industrial products with appealing performances [1-3]. Recently, rare earth based metallic glasses including cerium based ones (Ce-MGs) have been found to display thermoplastic behavior with very low glass transition temperature $\left(\mathrm{T}_{g}<373 \mathrm{~K}\right)$ and low Young's modulus in comparison with classical amorphous matter [4-7]. At room temperature, these

alloys are strong (elastic strain of $2 \%$ [4]) and brittle. In their supercooled-liquid state, these materials can however be repeatedly shaped into very fine structures down to the nanometer scale, of great interest for micro electro-mechanical systems or nanotechnology applications such as high-density data storage [8].

In this context, the structural [9-12], electronic [9, 13], elastic [7, 14-16] and thermal [17] properties of Ce-MGs have been extensively studied. Under pressure, an amorphousamorphous phase transition occurs from a low-density amorphous (LDA) phase to a highdensity amorphous (HDA) one. Indeed, previous x-ray diffraction measurements on Ce-MGs $[9,15,18]$ investigated such properties on polyamorphic transition in Ce-MGs upon high pressure. Such works have shown an important structural change under pressure which is reversible and exhibits hysteresis under decompression. Sheng's group [9, 10] has proposed a structural model for the LDA and HDA phases based on ab initio molecular dynamics simulations in which a splitting of the Ce-Ce nearest-neighbors distance is observed at the transition. The local structure at high pressure exhibits a double-shell feature, with an inner shell corresponding to a significant shorter bond compared to the low-pressure structure. In these calculations, based on the density functional theory with explicit local Coulomb repulsion $(\mathrm{DFT}+\mathrm{U})$, the LDA and HDA phases have been characterized by a localized and itinerant 4 f electron, respectively. Also using x-ray techniques, Duarte et al [15] reported a low- and high-density amorphous phases at different pressure ranges, with the occurrence of a hysteresis at the transition, and of an intermediate density region expected to coincide with a mixture of both phases, or with a new metastable phase. This last hypothesis has been recently reported by Luo et al [19], through an exploration of the LDA-HDA transformation path at the temperature of $390 \mathrm{~K}$.

In order to elucidate the nature of a polyamorphic transition and the corresponding 
phase diagram, one needs to extract the density of the compound as a function of pressure and temperature. However, all previous works have analyzed the experimental data using theoretical calculations or approximations in which the momentum transfer values for the first sharp diffraction peak (FSDP) can be roughly related to volume variation. In this work, we present a comprehensive experimental study of $C e_{69} A l_{10} C u_{20} C o_{1}$ bulk MG by means of angle dispersive x-ray diffraction performed up to $16 \mathrm{GPa}$ along two distinct isotherms (300 and $340 \mathrm{~K}$ ). The whole diffuse signals have been here processed following the procedure as detailed in the Appendix, devised by [20-22] and later by Morard et al [23]. In this way, we have been able to extract directly the structure factor $S(Q)$, the pair distribution $g(r)$, the atomic density $\rho$ and the compressibility as a function of pressure and temperature. These results are then discussed in the context of polyamorphism and in relation with the $\gamma \leftrightarrows$ $\alpha$ transition in pure cerium, as well as with the existence of a critical point in the phase diagram of Ce-MGs.

The paper is organized as follows. The experimental technique, together with the method of data analysis are presented in Sec. II and in the Appendix. The resulting high-pressure studies of the equation of state and polyamorphism on $\mathrm{Ce}_{69} A l_{10} C u_{20} \mathrm{Co}_{1}$ bulk MG are given in Sec. III (300 K isotherm) and in Sec. IV (340 K isotherm). The conclusion is drawn in Sec. V.

\section{EXPERIMENTAL TECHNIQUE AND ANALYSIS}

$\mathrm{Ce}_{69} \mathrm{Al}_{10} \mathrm{Cu}_{20} \mathrm{Co}_{1}$ bulk metallic glass was prepared in the form of thin ribbons (thickness $\approx 30 \mu \mathrm{m}$ ) using the single-roller melt-spinning technique. Its composition was confirmed by electron microprobe, scanning electron microscopy images showing uniform contrast. The glassy nature of the sample was verified by x-ray diffraction (no detectable Bragg peaks) and differential scanning calorimetry (presence of exothermic peak indicative of a glass transition temperature). This sample has been cut as few microns of dimensions and loaded in a rhenium gasket hole of $100 \mu \mathrm{m}$ diameter placed in a membrane diamond anvil cell (DAC), resistively heated and equipped with $300 \mu \mathrm{m}$ culet size diamonds. Neon was used as pressure transmitting medium. We used DACs able to independently control pressure and temperature without bringing the cell back to ambient conditions. The temperature was regulated using an electronic module and measured with an accuracy better than $1 \mathrm{~K}$ by two 


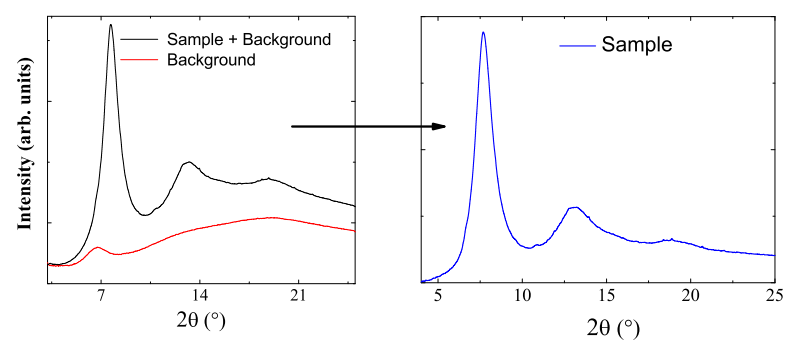

FIG. 1: (color online) (Left) Measured diffraction pattern $I_{\text {meas }}(Q)$ of $C e_{69} A l_{10} C u_{20} C o_{1}$ bulk metallic glass at $2.2 \mathrm{GPa}$ and $300 \mathrm{~K}$, including the background (red line, $I_{b k}(Q)$, mostly due to Compton signal from the diamonds here) which has been recorded at the same conditions in the experimental volume and beside the Ce-MG sample. (Right): Scattering signal of the sample $I_{S}(Q)$ (background removed).

K-type thermocouples glued one on each anvil. Pressures were determined from the ruby and $\mathrm{SrB}_{4} \mathrm{O}_{7}: \mathrm{Sm}^{2+}$ fluorescence lines before and after collection of each diffraction pattern. These two readings always provided both pressure and temperature values (corresponding uncertainties given by symbols sizes in all the following figures).

Angle dispersive x-ray diffraction experiments in diamond anvil cell were performed at ID27 beam-line at the European Synchrotron Radiation Facility (ESRF). Incident energy of $33 \mathrm{keV}$ was focused on the sample by using a focalized micrometric-sized x-ray beam. At each $(\mathrm{P}, \mathrm{T})$ point, a diffraction pattern of the background $I_{\mathrm{bk}}(Q)$ was recorded through the pressure transmitting medium (but out of the $C e_{69} A l_{10} C u_{20} C o_{1}$ glass sample), in order to be subtracted from the measured signals $I_{\text {meas }}(Q)$ (see Fig. 1).

The scattering signal from the $\mathrm{Ce}_{69} A l_{10} \mathrm{Cu}_{20} \mathrm{Co}_{1}$ bulk metallic glass sample only $I_{S}(Q)$ has been extracted from the measured signal after removing the background signal (using the classical Krogh-Moe Norman method [24]). Then, an iterative procedure has been applied to calculate the pair distribution function $g(r)$ as well as the density of the glassy alloy based on the Fourier treatment:

$$
g(r)=1+\frac{1}{2 \pi^{2} r \rho_{0}} \int_{0}^{\infty} Q(S(Q)-1) \sin (Q r) d Q=\frac{\rho(r)}{\rho_{0}} .
$$

The main steps of the corresponding numerical procedure are detailed in the Appendix, together with the basic assumptions and sources of uncertainties. 


\section{POLYAMORPHISM OF CERIUM-MG AT HIGH PRESSURE}

Using the previous experimental setup and analysis, the pressure evolution of the density of a prototypical Ce-based amorphous sample $\left(\mathrm{Ce}_{69} A l_{10} \mathrm{Cu}_{20} \mathrm{Co}_{1}\right)$ has been studied up to 16 GPa at ambient temperature. The occurrence of a pressure-induced phase transition from two glassy states can be markedly observed by a comparison of diffraction patterns $I_{\mathrm{S}}(Q)$ at, for example, 0.5 GPa and $11 \mathrm{GPa}$ (see Fig. 2). At low pressure, the FSDP is observed at $22.7 \mathrm{~nm}^{-1}$, followed by two other wider bumps around 38.7 and $55.2 \mathrm{~nm}^{-1}$ (respectively labeled a and b). Such patterns evolved at higher pressure, where the FSDP is shifted to $25.2 \mathrm{~nm}^{-1}$ with roughly the same intensity, whereas the $38.7 \mathrm{~nm}^{-1}$ bump intensity mainly weakens while shifting to higher Q-values. In parallel, a new structure at around $50 \mathrm{~nm}^{-1}$ is observed in the high pressure $(11 \mathrm{GPa}) S(Q)$ function. All these observations can also be found on the corresponding pressure evolution of the $g(r)$, represented in the right-hand side of Fig. 2.

The emergence of this new peak (labeled $c$ ) first demonstrates that a LDA-HDA transition occurs with local structure transformation probably related to the critical change of electronic properties of Ce atoms $[25,26]$. Secondly, the shift of the FSDP without intensity change shows that such transition does not significantly alter the mean distance order (taken, here, as the total coordination number). It suggests a transition related to a collapse of the main architecture of the first atomic shells, with shorter average atomic distance, apparent at high pressure. Note that this is a quite unusual behavior since in the majority of glassy oxides where polyamorphism has been demonstrated, the LDA-to-HDA transition goes along with a critical change of the mean distance order [11, 19].

In order to go further, an integration of the first $g(r)$ peak is known to give an information proportional to the total coordination number $N_{c}$ of the first atomic shell following the equation:

$$
N_{\mathrm{c}}=\int_{0}^{\mathrm{r}_{\mathrm{cut}}} 4 \pi r^{2} \rho_{0} g(r) d r
$$

where $r_{\text {cut }}$ is set as the location of the minimum at the right side of the first peak in the pair distribution function $g(r)$.

In the present work, and taken into account the experimental uncertainty, the evolution of $N_{c}$ is found to be pressure independent (inset of Fig. 3), with a typical value for a metallic alloy (here, $10 \pm 1$ ). In the same Figure, the pressure evolution of the relative density $\bar{\rho}=$ 

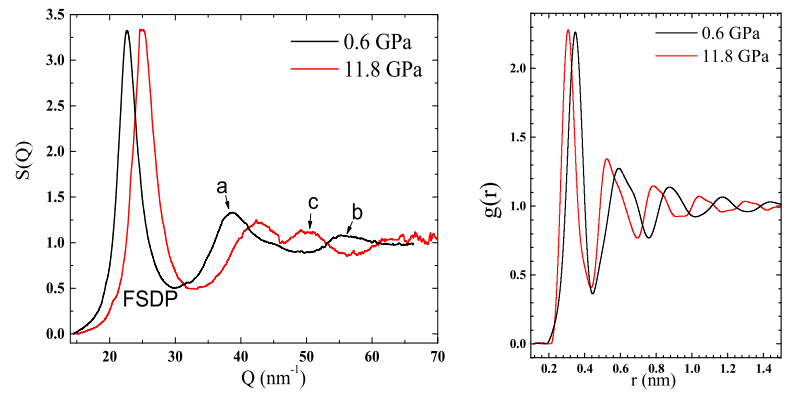

FIG. 2: Structure factor $S(Q)$ (left) and pair correlation function $g(r)$ (right) of $\mathrm{Ce}_{69} A l_{10} C u_{20} C o_{1}$ at $300 \mathrm{~K}$ and high pressure: $0.6 \mathrm{GPa}$ (black) and $11.8 \mathrm{GPa}$ (red).

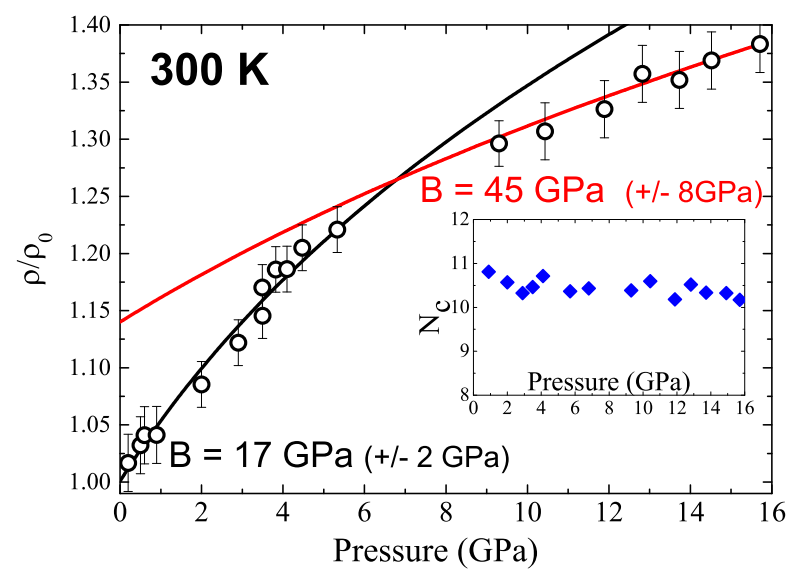

FIG. 3: Relative density of $C e_{69} A l_{10} C u_{20} C o_{1}$ as a function of pressure at $300 \mathrm{~K}$. Inset: pressure evolution of the corresponding total coordination numbers $\mathrm{N}_{c}$.

$\rho / \rho_{0}$ up to $16 \mathrm{GPa}$ is also represented. Moreover, note here that $\bar{\rho}$ has been defined as the number of atoms per volume units in proportion to the ambient conditions sample density (in this case $\rho_{0}$ has taken the value of $\rho_{0}{ }^{L D A}=38.2 \pm 2 \mathrm{at} / \mathrm{nm}^{3}$, as the sample is in the LDA state at ambient pressure).

A smooth but clear change in the pressure evolution of $\bar{\rho}$ is observed between 6 and 9 GPa, a finding which reinforces the previous arguments in favor of a LDA-HDA transition in $\mathrm{Ce}_{69} \mathrm{Al}_{10} \mathrm{Cu}_{20} \mathrm{Co}_{1}$. It must be emphasized that the iterative procedure to extract the density from the experimental signal did not converge in this pressure range. This could be due either to the presence of an intermediate local structure between the LDA and HDA phases [19], or to the coexistence between the two phases, as expected in a first-order phase 
transition.

In both low- and high-pressure pure phase domains, a Murnaghan equation of state has been used to fit the $\bar{\rho}(\mathrm{P})$ data, with $B^{\prime}$ fixed to 4 . It leads to $B_{0}{ }^{L D A}=17.4 \pm 2 \mathrm{GPa}\left(\rho_{0}{ }^{L D A}\right.$ $\left.=38.2 \pm 2 \mathrm{at} / \mathrm{nm}^{3}\right)$ and $B_{0}{ }^{H D A}=45.2 \pm 8 \mathrm{GPa}\left(\rho_{0}{ }^{H D A}=42.4 \pm 2 \mathrm{at} / \mathrm{nm}^{3}\right)$. Note here that the two Murnaghan equations of state give a density variation of about $12 \%$ at $7 \mathrm{GPa}$ (the average pressure along the transformation process), a discontinuity probably hidden experimentally by the kinetics of the transition between the different states.

\section{TEMPERATURE EFFECT ON THE PRESSURE INDUCED- POLYAMORPHISM OF CERIUM-MG}

While the polyamorphism in $C e_{69} A l_{10} C u_{20} C o_{1}$ has been demonstrated at ambient temperature in the previous section, the existence of such a feature along a higher-temperature isotherm stands as an open question. Firstly, because this sample belongs to the material family with long-range disordered structure, including amorphous and glasses, where the intriguing hypothesis of the two-scale theory, initially proposed by Rapoport [27], theoretically confirmed but never validated experimentally, is still debated. Secondly because this sample is cerium-based: a crucial consequence is that, as we recently observed by x-ray absorption experiments [25], the LDA-to-HDA transformation in cerium-based bulk metallic glasses can be tightly linked to the behavior of the single $4 f$ electron of $\mathrm{Ce}$, as for the $\gamma \rightleftarrows \alpha$ first-order transition in pure cerium. In the latter transition, studies under high pressure and high temperature have revealed the existence of a critical point $(\mathrm{CP})$ at high temperature in the solid phase [28, 29], driven by entropy difference, and probably also in the liquid state [30]. This CP plays an important role in the two-scale theory of polyamorphism despite it has never been observed [31]. Hence, studying the high-temperature behavior of solid Ce-BMG in order to search for the occurrence of such a CP in their phase diagram is an exciting area of research that should finally unravel the origin and mechanisms of polyamorphism.

Experimentally, the critical barrier is obviously related to the low kinetic stability of glasses which limits the temperature range of study. We performed a differential scanning calorimetry study of the present $\mathrm{Ce}_{69} \mathrm{Al}_{10} \mathrm{Cu}_{20} \mathrm{Co}_{1}$ glass alloy, which has revealed the presence of an exothermic peak (indicative of a glass transition temperature $T_{g}$ ) at $365 \mathrm{~K}$, and a crystallization temperature (at ambient conditions) of 416 K. Given these constraints, we 
also determined the equation of state of the sample at $340 \mathrm{~K}$ (well below the glass transition temperature), similarly to the previous angle dispersive x-ray diffraction experiments at 300 $\mathrm{K}$.

Typical structure factors $S(Q)$ and pair correlation function $g(r)$ at different pressure along the $340 \mathrm{~K}$ isotherms are shown in Fig. 4. Qualitatively, the same behavior as the ambient temperature one is here observed, with an obvious structural modification related to the appearance at about $3 \mathrm{GPa}$ of a peak at $55 \mathrm{~nm}^{-1}$ while the width and the intensity of the FSDP remain unchanged.
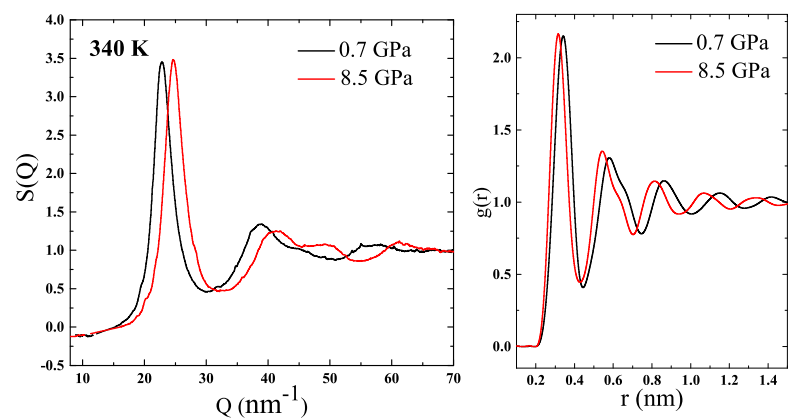

FIG. 4: Pressure evolution of the structure factor $S(Q)$ (left) and pair correlation function $g(r)$ (right) patterns of $\mathrm{Ce}_{69} \mathrm{Al}_{10} \mathrm{Cu}_{20} \mathrm{Co}_{1}$ along the $340 \mathrm{~K}$ isotherm.

Similarly, the evolution of total coordination number is found to be pressure independent, with $N_{c}=11 \pm 1$, while the relative density $\bar{\rho}=\rho / \rho_{0}$ up to 14 GPa needs to be fitted using two distinct Murnaghan equations of state (see Fig. 5) giving $B_{0}{ }^{L D A}=17.4 \pm 0.8 \mathrm{GPa}$ $\left(\rho_{0}{ }^{L D A}=37.9 \pm 2 \mathrm{at} / \mathrm{nm}^{3}\right)$ and $B_{0}{ }^{H D A}=53.2 \pm 13 \mathrm{GPa}\left(\rho_{0}{ }^{H D A}=42.4 \pm 2 \mathrm{at} / \mathrm{nm}^{3}\right)$. The density variation in the transition region (between 4 and $6 \mathrm{GPa}$ ) is here about $10 \%$, without significant differences if compared to what previously determined at $300 \mathrm{~K}$. Moreover, we do not observed here any abrupt and discontinuous variation of the FSDP intensity or position, in contrast to what was shown by Luo et al [19] at a temperature close but lower than the glass transition (in the latter study, a kink in the FSDP position and intensity was observed at about $5 \mathrm{GPa})$.

This disagreement could be explained by two factors. Firstly, the chemical composition of our sample is different (notably poorer in Co). Secondly, the temperature of our study is relatively lower (by 50 degrees), say not as close to $T_{g}$ as in the Luo et al study. However, the present work does not clearly discriminate a possible weak first-order nature as argued 


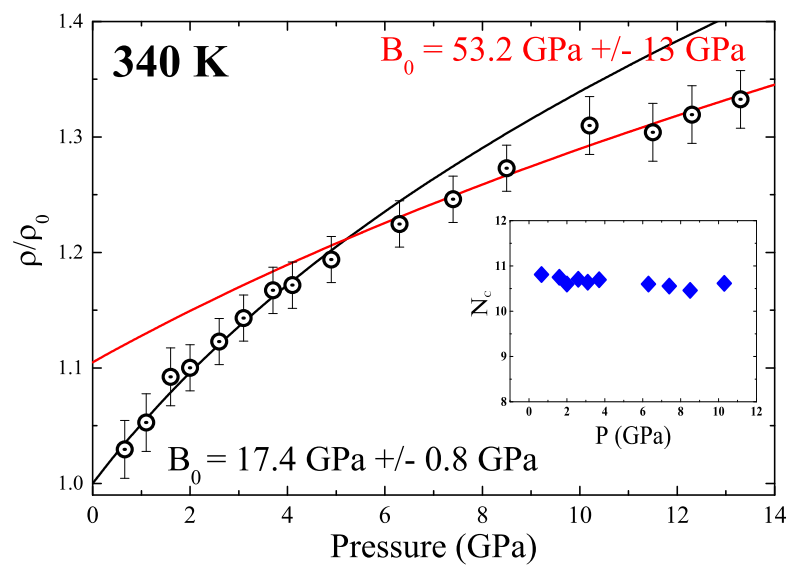

FIG. 5: Relative density as a function of pressure at $340 \mathrm{~K}$. Inset: pressure evolution of the corresponding total coordination numbers.

in Ref [19]. The discontinuous volume change measured in our work between 4 and 6 GPa at $340 \mathrm{~K}$, as it occurs for a first-order transformation, can actually be hidden by the coexistence of the low- and high-pressure phases between 4 and $6 \mathrm{GPa}$, or by the presence of an intermediate medium-density amorphous state [19].

Finally, the present data support the conclusion that the hypothetical existence of a critical point in Ce-MGs still has to be demonstrated, the temperature effect previously observed [25] on the electronic properties of such a type of sample being probably related to a classical kinetic effect.

\section{CONCLUSION}

The present angle dispersive x-ray diffraction study at high pressure and temperature on $C e_{69} A l_{10} C u_{20} C_{1}$ provides a direct experimental evidence that the LDA-to-HDA transformation occurs at high pressure and ambient temperature. Along an isotherm at higher temperature, a similar behavior is observed without any evidence of a clear first-order transition [19] nor of a critical point existence. In both cases (300 and $340 \mathrm{~K}$ ), the density pressure evolution is shown to be compatible with the general picture of structural rearrangement previously proposed by numerical simulations [9]. We observed in particular that the density change at the transition, roughly $10 \%$, occurs without variation of the total coordination number. As this polyamorphism in Ce-rich MG is known to be driven by a modification of 
Ce $4 \mathrm{f}$ states, our findings are in good agreement with the expected Ce-Ce bond shortening at the transition $[9,11]$. We emphasize that angle dispersive x-ray diffraction experiments only provide global information on the structure of the sample, and the role played by each type of atoms, particularly the cerium ones, cannot be discriminated here. However, it is interesting to highlight the connection between the LDA-to-HDA transformation in the Ce-MGs and the volume collapse transition in pristine Ce. In both cases, the density change is not accompanied by a rearrangement of the local geometry, nor by a modification of the coordination number, at variance with standard structural phase transitions. In a previous theoretical study of pure Ce [26] based on quantum Monte Carlo simulations, some of us pointed out that the Ce-Ce chemical bond variation, driven by strong local electron correlation and forbital hybridization, is key to understand the volume collapse. The corresponding phase transition is found already at zero temperature, even before the entropy effects dictated by the Kondo energy scale kick in, whose magnitude exponentially varies as a function of the f-electron hybridization [32]. In this paper we presented a compelling experimental evidence that also Ce-MGs, although very different from pure Ce, show a transition related to a significant volume variation. Volume collapse effects found in Ce-bearing compounds, such as Ce-MGs, are fascinating signatures of a general phenomenon, not specific to pristine Ce or Ce-alloys crystals, which calls for a further theoretical and experimental understanding.

\section{APPENDIX: ANALYSIS OF DIFFUSE SIGNAL FROM BULK METALLIC GLASSES}

\section{A. Theoretical background}

Within the general frame of a polyatomic compound, the spherically averaged coherent x-ray scattering from disordered atoms can be written as:

$$
I_{c o h}(Q)=N \sum_{p} f_{p}^{2}(Q)+\sum_{m} \sum_{n \neq m} f_{m}(Q) f_{n}(Q) \frac{\sin Q r_{m n}}{Q r_{m n}},
$$

where $N$ is the total number of compositional (or "elementary") units, $m$ and $n$ are the atoms in the entire volume of the x-ray-illuminated sample (labeled as $\Omega$ ) whereas $p$ defines atoms within an elementary unit volume $\mathrm{dV}, f_{n}$ the atomic form factor of the $n$-th atom, and $Q=4 \pi \sin \theta / \lambda$ the scattering momentum. 
In the following, the average atomic distribution $\rho_{p q}(\mathrm{r})$ will be defined in such a way that the quantity $\rho_{p q}(\mathrm{r}) \mathrm{dV} \equiv<\rho_{p}\left(\mathrm{r}_{p q}\right) \mathrm{dV} \mathrm{V}_{q}>_{\Omega}$ is the number of $q$ atoms in an elementary volume $d V$ at a distance $r$ of a $p$-type atom taken as the center of the chemical unit. Then, Eq. 3 can be rearranged as an integral over the x-ray-illuminated sample volume $\Omega$ :

$$
I_{c o h}(Q)=N \sum_{p} f_{p}^{2}(Q)+N \sum_{p} \sum_{q} \int_{\Omega} f_{p}(Q) f_{q}(Q) \rho_{p q}(r) \frac{\sin Q r}{Q r} d V .
$$

Following the Faber-Ziman method [33], the previous $I_{c o h}(Q)$ can also be expressed as:

$$
I_{c o h}(Q)=\left\langle f^{2}\right\rangle+\langle f\rangle^{2} \int_{0}^{\infty} 4 \pi r^{2}\left(\rho(r)-\rho_{0}\right) \frac{\sin Q r}{Q r} d r
$$

where $\rho_{0}$ is the atomic density of the sample and $\rho(r)$ is the effective density function defined as:

$$
\rho(r)=\frac{\sum_{m} \sum_{n} \rho_{m n}(r) X_{m}\left\langle f_{m}(Q)\right\rangle_{Q} X_{n}\left\langle f_{n}(Q)\right\rangle_{Q}}{\sum_{m} \sum_{n}\left\langle X_{m} f_{m}(Q) X_{n} f_{n}(Q)\right\rangle_{Q}}
$$

where $\mathrm{X}_{i}$ is the occurrence of $i$ atoms within the sample volume, and $\langle\ldots\rangle_{Q}$ denotes the average over the $Q$ range at which the x-ray scattering experiment is performed.

The intermediate function $\langle f\rangle^{2}$ and $\left\langle f^{2}\right\rangle$ are respectively defined as:

$$
\langle f\rangle^{2}=N \sum_{p} \sum_{q} f_{p}(Q) f_{q}(Q)=\sum_{m} \sum_{n} X_{m} f_{m}(Q) X_{n} f_{n}(Q)
$$

and

$$
\left\langle f^{2}\right\rangle=N \sum_{p} f_{p}^{2}(Q)=\sum_{n} X_{n} f_{n}^{2}(Q),
$$

with $p$ and $q$ in the above equations running on the elementary unit, while $n$ and $m$ run over the whole sample $\Omega$.

The structure factor normalized to unity (here called Faber-Ziman structure factor $S_{F Z}$ ) can then be expressed as:

$$
\begin{array}{r}
S_{F Z}(Q)=\frac{I_{c o h}(Q)-\left(\left\langle f^{2}\right\rangle-\langle f\rangle^{2}\right)}{\langle f\rangle^{2}} \\
=1+\int_{0}^{\infty} 4 \pi r^{2}\left(\rho(r)-\rho_{0}\right) \frac{\sin Q r}{Q r} d r .
\end{array}
$$

A Fourier transform of the previous equation thus leads to the experimental distribution function $F(r)$ :

$$
F(r)=4 \pi r\left(\rho(r)-\rho_{0}\right)=\frac{2}{\pi} \int_{0}^{Q_{\max }} Q\left(S_{F Z}(Q)-1\right) \sin (Q r) d Q,
$$

from where the pair distribution function $g(r)$ can be extracted as:

$$
g(r)=\frac{\rho(r)}{\rho_{0}} .
$$




\section{B. Krogh-Moe Norman normalization}

The intensity of the signal scattered from the sample $I_{S}(\mathrm{Q})$ is calculated from the measured intensity $I_{m e s}(Q)$ as the following:

$$
I_{S}(Q)=I_{m e s}(Q)-b I_{b k}(Q),
$$

with $I_{b k}$ the intensity of the signal coming from the sample environment (anvils, pressure transmitting medium, etc.). This signal, here acquired under the same pressure and temperature conditions but without scattering from the Ce-MG, is adjusted by a scale factor $b$ to account for the slight difference in experimental conditions (coming from, as example, the thickness of pressure transmitting medium or the pressure gradient within the experimental volume).

Following the method developed by Krogh-Moe [24] and Norman [34], $I_{S}(Q)$ is then converted into the normalized structure factor units:

$$
\begin{aligned}
S_{F Z}(Q) & =\frac{I_{c o h}-\left(\left\langle f^{2}\right\rangle-\langle f\rangle^{2}\right)}{\langle f\rangle^{2}} \\
& =\frac{\left(\alpha I_{S}(Q)-\sum I_{\text {incoh }}(Q)\right)-\left(\left\langle f^{2}\right\rangle-\langle f\rangle^{2}\right)}{\langle f\rangle^{2}},
\end{aligned}
$$

where the normalization factor $\alpha$ is:

$$
\alpha=\frac{-2 \pi^{2} \rho_{0}+\int_{0}^{Q_{\max }} \frac{\sum I_{\text {incoh }}(Q)+\left\langle f^{2}\right\rangle}{\langle f\rangle^{2}} Q^{2} d Q}{\int_{0}^{Q_{\max }} \frac{Q^{2} I_{S}(Q)}{\langle f\rangle^{2}} d Q} .
$$

In the above equation, $Q_{\max }$ is the maximum scattering momentum, typically around $70-80$ $\mathrm{nm} .^{-1}$ for DAC experiments, and $\sum I_{\text {incoh }}(Q)$ is the sum of the incoherent scattering signals from the sample. The effect of the limited $Q$ range, in particular on the density, will be discussed later. Note that the $Q$ range used in the present study is suitable for the study of amorphous compounds and does not lead to spurious peaks in the $g(r)$ [35].

\section{Iterative procedure}

To extract the sample density $\rho_{0}$, an numerical iterative procedure with basic assumptions has to be carried out in order to minimize the error in the determination of $g(r)$.

The first step here is to calculate the experimental distribution function $F(r)$ using equation 10 with given $\rho_{0}$ et $b$ values, as starting guess. 
In a second step, a minimal distance $\mathrm{r}_{\min }$ is defined in such a way that the distance between 0 and $\mathrm{r}_{\min }$ represents the largest distance where no atom can be found. For $r<r_{\min }$, no atom can be present, i.e. $\rho(r)=0$, so that no oscillation should be observed in the $g(r)$ function. As a consequence, we also have a particular distribution function $F(r)$ which is $F\left(r<r_{\min }\right)=-4 \pi r \rho_{0}$.

Third, one can calculate the difference between model and real data $\Delta F_{0}(r)$ (where index 0 represents the value of the function before the first iteration) such as:

$$
\Delta F_{0}(r)=-F_{0}(r)-4 \pi r \rho_{0}, \quad \text { for } 0<r<r_{\min }
$$

$\Delta F_{0}(\mathrm{r})$ thus represents the error on the calculation of $F(r)$ before the first iteration. Two types of systematic errors can usually take place here.

The first one is related to the determination of the normalization factor $\alpha$, mainly affected by the experimental limited $Q$ range $\left(Q_{\max }\right.$ in Eq. 14). Loss of information present at large $Q$ obviously affects the values of density determined using the hereby procedure. This source of error is however quite small since the structure factor of glasses naturally damps quickly with increasing $Q$, so that the main part of the information is comprised in the first two oscillations.

Beside this truncation effect, another source of uncertainty comes from the determination of the $b$ scale factor in the background subtraction. In the present work, the background was substantial which did not allow for a stringent fitting procedure of $b$. We thus fixed it manually, using exactly the same procedure for each pressure point, so that no relative error are expected to occur in the final determination of the pressure evolution of the sample density.

At this point, following the work of Kaplow et al. [36], the iterative procedure can be written as:

$$
\begin{aligned}
i(Q) & =S(Q)-1, \\
i(Q) & =i_{0}(Q)+\Delta i(Q), \\
\alpha & =\alpha_{0}(1+\Delta \alpha),
\end{aligned}
$$

with $\Delta i(Q)$ et $\alpha_{0} \Delta \alpha$ the error on the $i$ et $\alpha$ variables, respectively. We emphasize that small variations of the normalization factor $\alpha$ could lead through this iterative scheme to large variations in $S(Q)$, and so in $g(r)$. 
It goes:

$$
\begin{array}{r}
Q \Delta i(Q)=Q \Delta \alpha S_{0}(Q)=\Delta \alpha\left(Q i_{0}(Q)+Q\right) \\
=\Delta \alpha Q i_{0}(Q)+\Delta \alpha Q,
\end{array}
$$

where the first term is a scaling factor for $S(Q)$, and the second one, called ramp term, leads to large oscillation in the low $r$ region of the $g(r)$ [20]. The basic assumption is then to assert that the ramp term is the main source of $\Delta F_{0}(r)$, which leads to the following equation, at the heart of the iterative process:

$$
\Delta \alpha Q=\int_{0}^{r_{\min }} \Delta F_{0}(r) \sin (Q r) d r .
$$

$\Delta \alpha$ is directly determined by the experimental $\Delta F(r)$. Using the combination of Eq. 19 and Eq.. 20, the error on $S(Q)$ coming from $\Delta \alpha$ is determined, and a new structure factor is obtained through:

$$
S_{1}(Q)=S_{0}(Q)\left(1-\frac{1}{Q} \int_{0}^{r_{\min }} \Delta F_{0}(r) \sin (Q r) d r\right) .
$$

The iterative process is then built by re-injecting $S_{1}(Q)$ into Eq. 10 to obtain $F_{1}(r)$ and recalculate $\Delta F_{1}(r)$.

In the present work, typically five iterative steps were necessary to converge. The corresponding fifth $\chi^{2}$ is thus calculated as:

$$
\chi^{2}\left(\rho_{0}, b\right) \equiv \int_{0}^{r_{\min }}\left(\Delta F_{5}(r)\right)^{2} d r .
$$

The above function exhibits one well defined minimum [20], here determined through a the simplex minimization procedure method [37] which allows to determine the unique $\left(\rho_{0}, b\right)$ couple at convergence.

\section{Acknowledgments}

We acknowledge G. Le Marchand and P. Munsch (IMPMC) for the DAC preparation. We also thank G. Weck for fruitful discussions.

[1] W. Wang, Advanced Materials 21, 4524 (2009). 
[2] J. C. Huang, J. P. Chu, and J. S. C. Jang, Intermetallics 17, 973 (2009).

[3] E. Lutanie, Materials Today 12, 12 (2009).

[4] B. Zhang, D. Q. Zhao, M. X. Pan, W. H. Wang, and A. L. Greer, Physical Review Letters 94, $205502(2005)$.

[5] J. Li, J. Wang, X. Liu, K. Zhao, B. Zhang, H. Bai, M. Pan, and W. Wang, Science ChinaPhysics mechanics and Astronomy 53, 409 (2010).

[6] B. Zhang, D. Zhao, M. Pan, R. Wang, and W. Wang, Journal of Non-Crystalline Solids 352, 5687 (2006).

[7] D. Ma, A. D. Stoica, X.-L. Wang, Z. P. Lu, B. Clausen, and D. W. Brown, Phys. Rev. Lett. 108, 085501 (2012).

[8] G. Marsh, Materials Today 6, 38 (2003).

[9] H. W. Sheng, H. Z. Liu, Y. Q. Cheng, J. Wen, P. L. Lee, W. K. Luo, S. D. Shastri, and E. Ma, Nature Materials 6, 192 (2007).

[10] H. W. Sheng, W. K. Luo, F. M. Alamgir, J. M. Bai, and E. Ma, Nature 439, 419 (2006).

[11] Q. Zeng, H. Sheng, Y. Ding, L. Wang, W. Yang, J.-Z. Jiang, W. L. Mao, and H.-K. Mao, Science 332, 1404 (2011).

[12] Q. Zeng, Y. Ding, W. Mao, W. Luo, A. Blomqvist, R. Ahuja, W. Yang, J. Shu, S. Sinogeikin, Y. Meng, D. Brewe, J. Jiang, and H. Mao, PNAS 106, 2515 (2009).

[13] Q. S. Zeng, C. R. Rotundu, W. L. Mao, J. H. Dai, Y. M. Xiao, P. Chow, X. J. Chen, C. L. Qin, H.-k. Mao, and J. Z. Jiang, Journal of Applied Physics 109, 113716 (2011).

[14] B. Zhang, M. Pan, D. Zhao, and W. Wang, Applied Physics Letters 85, 61 (2004).

[15] M. Duarte, P. Bruna, E. Pineda, D. Crespo, G. Garbarino, R. Verbeni, K. Zhao, W. Wang, A. Romero, and J. Serrano, Physical Review B 84 (2011).

[16] Q. Zeng, Y. Li, C. Feng, P. Liermann, M. Somayazulu, G. Shen, H. Mao, R. Yang, J. Liu, T. Hu, and J. Jiang, PNAS 104, 13565 (2007).

[17] Q. Zeng, Y. Fang, H. Lou, Y. Gong, X. Wang, K. Yang, A. Li, S. Yan, C. Lathe, F. Wu, X. Yu, and J. Jiang, Journal of Physics and Condensed Matter 22 (2010).

[18] Q. Zeng, V. Struzhkin, Y. Fang, C. Gao, H. Luo, X. Wang, C. Lathe, W. Mao, F. Wu, H.-K. Mao, and J. Jiang, Physical Review B 82 (2010).

[19] Q. Luo, G. Garbarino, B. Sun, D. Fan, Y. Zhang, Z. Wang, Y. Sun, J. Jiao, X. Li, P. Li, et al., Nature communications 6 (2015). 
[20] J. Eggert, G. Weck, P. Loubeyre, and M. Mezouar, Physical Review B 65 (2002).

[21] T. Egami and S. J. L. Billinge, Underneath the Bragg Peaks: Structural Analysis of Complex Materials, edited by U. Pergamon Press, Oxford (2003).

[22] C. E. White, Zeitschrift für Kristallographie Crystalline Materials 227, 304 (2012).

[23] G. Morard, G. Garbarino, D. Antonangeli, D. Andrault, N. Guignot, J. Siebert, M. Roberge, E. Boulard, A. Lincot, A. Denoeud, and S. Petitgirard, High Pressure Research 34, 9 (2014).

[24] J. Krogh-Moe, Acta Crystallographica 9, 951 (1956).

[25] L. Belhadi, F. Decremps, S. Pascarelli, L. Cormier, Y. Le Godec, S. Gorsse, F. Baudelet, C. Marini, and G. Garbarino, Applied Physics Letters 103, 111905 (2015).

[26] N. Devaux, M. Casula, F. Decremps, , and S. Sorella, Physical Review B 91, 081101 (2015).

[27] E. Rapoport, The Journal of Chemical Physics 46, 2891 (1967).

[28] M. Lipp, D. Jackson, H. Cynn, C. Aracne, W. Evans, and A. McMahan, Physical Review Letters 101 (2008).

[29] F. Decremps, L. Belhadi, D. Farber, K. Moore, F. Occelli, M. Gauthier, A. Polian, D. Antonangeli, C. Aracne-Ruddle, and B. Amadon, Physical Review Letters 106 (2011).

[30] A. Cadien, Q. Y. Hu, Y. Meng, Y. Q. Cheng, M. W. Chen, J. F. Shu, H. K. Mao, and H. W. Sheng, Phys. Rev. Lett. 110, 125503 (2013).

[31] P. McMillan, M. Wilson, M. Wilding, D. Daisenberger, M. Mezouar, and G. Greaves, Journal of physics condensed matter 19 (2007).

[32] B. Amadon and A. Gerossier, Physical Review B 91, 161103 (2015).

[33] Z. Faber and J. M. Ziman, Philos. Mag. 11 (1965).

[34] N. Norman, Acta Crystallographica 10, 370 (1957).

[35] Y. Waseda, The structure of non-crystalline materials (McGraw-Hill International Book Co., 1980).

[36] R. Kaplow, S. L. Strong, and B. L. Averbach, Phys. Rev. 138, A1336 (1965).

[37] J. Nelder and R. Mead, Computer Journal 4, 308 (1965). 